\title{
Inclusión Gerenciada y Escolarizaciones Low Cost. Una Analítica de Episodios en Escuelas Estatales del Sur Global
}

\section{Managed Inclusion and Low Cost Schooling. Episodes Analysis in State Schools in the Global South}

\author{
$\mathrm{M}^{\mathrm{a}}$ Cecilia Bocchio * \\ Cintia Schwamberger \\ Julieta Armella \\ Silvia Grinberg
}

Consejo Nacional de Investigaciones Científicas y Técnicas, Argentina

\begin{abstract}
En este artículo, a través de resultados de una investigación de tipo cualitativa, desarrollamos una lectura de las dinámicas de la inclusión educativa en escuelas emplazadas en contextos de pobreza urbana en dos grandes conglomerados de Argentina (Región Metropolitana de Buenos Aires y ciudad de Córdoba). Como estrategia metodológica recuperamos, desde un enfoque etnográfico, la noción de episodio a los efectos de construir una analítica de la vida de las escuelas donde la inclusión se produce de modo low cost. En la hipótesis proponemos que la inclusión educativa en escuelas caracterizadas por múltiples desigualdades deviene inclusión gerenciada, esto es, descansa en la capacidad de individuos e instituciones que se vuelven sujetos y objetos de aquello que, se supone, debe resolver el Estado. Se trata de un clivaje de la inclusión desde el cual es imperativo analizar tanto las lógicas que operan en detrimento del derecho a la educación como sus efectos en la precarización de quienes hacen escuela cotidianamente.
\end{abstract}

Descriptores: Desigualdad social; Cotidianeidad escolar; Escuela; Autogestión; Inclusión.

In this article, through results of a qualitative research, we develop an interpretation of the dynamics of educational inclusion in schools located in contexts of urban poverty in two large conglomerates of Argentina (Metropolitan Region of Buenos Aires and Córdoba city). As methodological strategy, from an ethnographic approach, we recover the notion of episode in order to construct an analysis of school's life where inclusion occurs in a low-cost way. As a hypothesis, we propose that educational inclusion in schools characterized by multiple inequalities becomes managed inclusion, that means, it rests on the capacity of individuals and institutions to become subjects and objects of what the State is supposed to solve. This is a cleavage of inclusion from which it is imperative to analyze both the logics that operate in detriment of the right to education and its effects on the precariousness of those who makes school daily.

Keywords: Social inequality; School life; Schools; Self-management; Inclusion. 


\section{Introducción ${ }^{1}$}

La inclusión, tanto en lo que refiere al diseño y desarrollo de políticas públicas como a la investigación, ha ido ganando terreno desde finales del siglo XX. Asociada, en sus inicios, a la educación especial (Infante, 2010) sus usos se fueron generalizando como una noción de mayor amplitud que refiere a una serie de prácticas que buscan revertir la exclusión. Por tanto, entendemos a la inclusión como el proceso por el cual los sistemas educativos, la cultura y la sociedad en general deben realizar transformaciones sistémicas a fin de eliminar las distintas formas de discriminación. En lo que refiere a la educación, tal como enuncia Etxeberria (2018), la inclusión se convierte en el reverso ético de la exclusión tendiente a garantizar el acceso, la participación y logros de todos los y las estudiantes, independientemente de su situación de clase, género, etnia y discapacidad, entre otros, con el objetivo fundamental de alcanzar una sociedad más justa e igualitaria.

La UNESCO (2017) en su cuarto Objetivo de Desarrollo Sostenible establece como prioridad "garantizar una educación inclusiva, equitativa y de calidad y promover oportunidades de aprendizaje durante toda la vida para todos". Ello implica, transformar la cultura, las políticas y las prácticas de las escuelas para atender la diversidad de necesidades de todos/as, desde una perspectiva derechos y en un proceso de constante transformación tanto educativo como social (Sandoval, Simón y Echeita, 2019).

La inclusión aparece, entonces, en los textos políticos que regulan la escolaridad una y otra vez, como un futuro más o menos próximo. Y de este modo pareciera enunciar eso que aún no ocurrió y que se propone como centro en la agenda de las políticas públicas. Particularmente, se torna veloz y se profundiza a partir de las diversas crisis que nuestras sociedades van sufriendo. Como Echeita (2016) lo afirma, la noción de inclusión se volvió un concepto escurridizo y de difícil aplicación, dado que es un concepto que paradójicamente cobra centralidad en un escenario que, desde finales del siglo XX, arroja a cada vez más vastos sectores de la población fuera del mercado formal del trabajo, viviendo en los bordes de las metrópolis, especialmente en el sur global (Grinberg, 2019). Para Bauman (2013) la glocalización y lo glocal señalan procesos sobre los cuales es tan difícil como desaconsejable minimizar el papel global que lo local desempeña en la puesta en funcionamiento y en la construcción de preceptos culturales necesarios para abordar los desafíos planteados por las desigualdades que la "interdependencia global" ha generado. En este contexto, la escuela ha sido y es parte clave de esa trama en tanto se espera que allí ocurran los procesos de inclusión, justamente, de una población que no deja de luchar contra la exclusión. Así, la noción de inclusión se ha vuelto la contracara de una sociedad que, como señalara Foucault (2013), ha renunciado al pleno empleo y que, en su seno, produce a diario una población liminar o flotante.

En este marco de debates, abordamos los procesos cotidianos de inclusión en el devenir de la vida escolar. Al respecto, proponemos la noción inclusión gerenciada como un modo de acercarnos a la descripción y comprensión de políticas de escolarización que, mientras reclaman inclusión, trasladan la responsabilidad de tales procesos a individuos e instituciones. Siguiendo esta línea argumentativa advertimos, una analogía entre lo low cost, es decir el bajo coste empresarial -que se corresponde con un modelo de negocios

\footnotetext{
${ }^{1}$ El trabajo de campo recuperado en este artículo se enmarca en dos proyectos de investigación financiados por el Consejo Nacional de Investigaciones. Científicas y Técnicas (CONICET).
} 
basado en la eficiencia de los recursos invertidos para reducir el costo del servicio y masificar los niveles de consumo (Vals, 2013)- y las lógicas de gerenciamiento de las escuelas emplazadas en contextos de pobreza urbana (Grinberg, 2020), donde las prácticas de inclusión devienen en escolarizaciones low cost (Bocchio, 2019). Es decir, se convierten en escolarizaciones que dependen de la maximización de los siempre insuficientes recursos disponibles en las escuelas, particularmente los materiales y humanos, bajo lógicas de rendimiento y agencia de los sujetos que participan. Así, la inclusión se propone como imperativo que atraviesa, de diferentes formas, la subjetividad de quienes están en la escuela.

A la luz de los aportes del campo de la gubernamentalidad, consideramos que estas escolarizaciones constituyen modos cotidianos de un hacer escuela que habilitan a que la inclusión ocurra siempre que las instituciones pueden autogestionar(se) a partir de una materialidad escolar cuyo signo es la fragilidad, cuando no la ausencia total de recursos. El bajo costo de escolarizar a quienes deben, además, ser incluidos, constituye una de las formas de las políticas de inclusión que trasladan a los sujetos, instituciones o barrios la responsabilidad de la gestión, sin los recursos para hacerlo. Así, al tiempo que la exclusión se vuelve la práctica más habitual del capitalismo flexible (Bauman, 2014), la inclusión deviene un imperativo político tendiente a revertir tales procesos. Mientras tanto las condiciones cotidianas de la escolaridad no hacen más que profundizar aquello que se espera resolver. Aun así, y como lo planteamos a través de la discusión del material empírico, esas mismas escuelas sino consiguen revertir la exclusión, al menos, logran torcerle el brazo.

Presentamos una analítica de episodios ocurridos en tres escuelas públicas de gestión estatal, dos de ellas de educación secundaria común y una de educación especial; dos ubicadas en la región metropolitana de Buenos Aires y una en la ciudad de Córdoba. Las tres comparten, entre otras particularidades, el hecho de estar emplazadas en aquellos espacios urbanos que conjugan pobreza y degradación ambiental. Esta particular situación nos adentra en detalle en lo que hace a la materialidad de las prácticas de in-exclusión (Lopes Fabris, 2016).

En esta línea, planteamos como hipótesis primaria que la inclusión educativa en escuelas signadas por múltiples desigualdades (pobreza urbana, condición social y económica de los estudiantes y sus familias, inestabilidad laboral del profesorado, precarias condiciones edilicias y de dotación de recursos, paupérrimos programas de alimentación en espacio escolar, etc.) ha devenido en una tarea, otra más, a ser gestionada por sus directivos y docentes que supone una tenacidad y un optimismo, muchas veces, casi patológico (Duk y Murillo, 2018). En consecuencia, y como hipótesis secundaria, planteamos que entre las dinámicas de inclusión-exclusión, se promueven procesos y prácticas de autogestión y agencia (Grinberg, 2019, 2020) que acontecen sin contar con los recursos ni las condiciones para garantizar la inclusión. Así, la noción de inclusión gerenciada la proponemos como un modo de describir un clivaje de la inclusión que nos interpela tanto en el análisis de los contextos escolares singulares/locales, signados por la precaridad (Butler, 2010), como de los efectos de subjetivación y precarización de quienes hacen escuela cotidianamente. Entendemos que tales efectos constituyen también una realidad global que signa nuestro tiempo. 


\section{Clivajes de la inclusión educativa. De la in/exclusión a la inclusión gerenciada}

Argentina legisla la inclusión educativa en el marco de la Ley de Educación Nacional $\mathrm{N}^{\circ}$ 26.206 (LEN) del año 2006. Su principio rector se vuelve, así, garantizar la educación como derecho bajo la premisa de la eliminación de todo tipo de discriminación y promoviendo políticas de reconocimiento y redistribución de recursos. A partir de ello, surgen diversas resoluciones que van adecuando, entre la normativa nacional y los postulados internacionales, los objetivos de garantizar el derecho a una educación inclusiva, equitativa y de calidad para todos los niños, niñas y adolescentes sin motivos de exclusión. Debates promovidos en las últimas dos décadas (Dussel, 2004; Kaplan, 2017; Kessler, 2014) visibilizan la intersección que existe entre diversas desigualdades y ponen en tensión aquello que se advertía sobre los riesgos de quedar a la deriva de la exclusión. En ese sentido proponemos que las políticas y acciones de inclusión no pueden ser pensadas de manera lineal, sino que requieren ser comprendidas en su territorio, en cada comunidad de la que son parte.

Retomando a Esposito (2005), la complejidad inherente a estas dinámicas se produce en un interjuego donde la protección de la vida y su desamparo se combaten no tanto en la negación sino en el rodeo. Así, señala:

La figura dialéctica que de este modo se bosqueja es la de inclusión excluyente o de la
exclusión mediante la inclusión. El veneno es vencido por el organismo no cuando es
expulsado fuera de él, sino cuando de algún modo llega a formar parte de este. Ta se
decía: más que a una afirmación, la lógica inmunitaria remite a una no-negación, a
la negación de una negación. Lo negativo no sólo sobrevive a su cura, sino que
constituye la condición de eficacia de ésta... un negativo menor destinado a bloquear
el mayor peso, pero en el interior del mismo lenguaje. (p.18)

De forma que, tal como lo discutiremos más adelante, la tensión inclusión-exclusión en las dinámicas cotidianas de la escolaridad coloca a las escuelas ante la imperiosa necesidad de gestionar aquello que sistémicamente sólo puede ser resuelto por fuera de ellas -llámese mantenimiento edilicio o elementos didácticos- y con recursos que la institución debe autogestionar/generar. A este proceso y sus modulaciones lo denominamos inclusión gerenciada. Declinación que refiere a la forma propia del negativo menor al que refiere Esposito (2005). Proponemos que también allí se produce un plus que es objeto de atención: la escuela en los barrios más empobrecidos del sur global es y se hace en estas tensiones y en ellas se producen un sinnúmero de luchas y fugas sobre las que es clave detener la mirada. No se trata solo de un padecer sino de sujetos que pujan y luchan en tanto la inclusión-exclusión no deja de ser parte de la gubernamentalidad contemporánea.

Los procesos de inclusión-exclusión en el sur global constituyen engranajes claves de la conducción de las conductas. Consideramos que la escuela ocupa un lugar central en la comprensión de las dinámicas que Bauman (2014) denomina glocales, así, la regulación de la escolarización de quienes viven en la pobreza urbana es un proceso que acontece situada y localizadamente, pero que también está inserta en dinámicas globales que toman lugar, con diferentes matices, en las metrópolis más desiguales redefiniendo y validando nuevos y a veces escandalosos modos y condiciones de escolarización.

En ese marco y retomando a Foucault (2013) el (neo)liberalismo se vuelve un conjunto de prácticas que configuran formas de vida, cada vez más regidas por principios de mercado y autorreflexión, donde los procesos de enseñanza y aprendizaje deben ser permanentes a 
la vez que centrados en el individuo. Es, desde esta perspectiva, que Lopes y Fabris (2016) señalan que no existe forma de quedar por fuera de la política neoliberal, dado que las prácticas de gobierno y de subjetivación (en tanto acción del sujeto sobre sí mismo) son modificadas, promoviendo la emergencia de nuevas formas de gubernamentalidad signadas por las contingencias políticas, sociales, económicas, educacionales. Las investigadoras señalan que en la actualidad esos sentidos y fuerzas definen una subjetividad inclusiva lo que significa "desarrollar una forma de ser que lleva a los individuos a ocuparse de los otros, capitalizando para sí mismos condiciones que los hacen más competitivos" (Lopes y Fabris, 2016, p. 67).

De este modo, directivos y docentes se ocupan de la vida de los muchos otros. Gestionar la inclusión se vuelve un trabajo necesario, obligatorio para conseguir ser escuela, para competir en el mercado escolar por la matrícula y también para evitar, sino ralentizar dinámicas de exclusión que ponen de manifiesto las lógicas de vulnerabilidad, precarización y/o marginación que afectan tanto a quienes la administran a nivel de las escuelas (directivos y docentes) como a quienes la viven a diario (estudiantes y familias).

Los estudios sobre gubernamentalidad (Grinberg, 2020; Veiga-Neto y Lopes, 2012) en su intersección con la educación nos brindan elementos para entender cómo las lógicas gerenciales atraviesan la vida social y proponen determinados mecanismos y tecnologías que irrumpen, se solapan, contraponen y/o yuxtaponen en la escuela. Ello entendiendo que no se trata de modos del arte de gobernar que se suceden, evolucionan o progresan, sino que son más bien líneas de fuerza, prácticas sedimentadas, contraconductas y formaciones donde enunciados o tecnologías que en una determinada formación histórica constituyen la verdad de una época, en otra se vuelven saberes menores (Foucault, 2013). Así, proponemos que para directivos y docentes la gestión de los recursos, de la incertidumbre y de la masividad de una escuela se transforma en un arte colectivo y cotidiano que los coloca ante dinámicas permanentes de autogestión y autoayuda. Afín a las lógicas neoliberales se involucran prácticas de gobierno de la población donde la política es eficiente si sirve para, al menos, declamar la democratización de la escuela secundaria y pretender alcanzar buenos resultados con una inversión mínima, una inclusión low cost. En el territorio escolar los textos políticos enarbolan la inclusión como bandera, mientras se forma a los directivos y docentes en el convencimiento de que el esfuerzo por la comunidad escolar vale la pena, una lógica de "gobierno a través de la comunidad" (Rose, 2007) como tecnología que propone diversas estrategias para promover la lealtad entre individuos y comunidades para con proyectos de regulación, reforma o movilización social.

Ahora bien, en el día a día escolar, directivos y docentes comprueban que si no lo hacen ellos nadie lo hará. Así, inmersos en las lógicas de la "Nueva Gestión Pública" (NGP) la regulación moral de las conductas se apropia de la vida escolar dejando a docentes y directivos sobrecargados de responsabilidades, pero sin los recursos para hacerles frente, en un particular estado que combina agobio y empuje permanente (Bocchio y Grinberg, 2019). En suma, en una conquista por la inclusión.

Como ya adelantamos, proponemos aquí la noción de inclusión gerenciada para referir al conjunto de tensiones y acciones que los sujetos (auto)gestionan a nivel de las escuelas, procurando garantizar las condiciones -no garantizadas desde el Estado- para hacer frente a desigualdades sociales y territoriales que signan la escolarización de quienes históricamente fueron excluidos del sistema educativo. Aquí nos ocupamos de describir y 
problematizar acciones a los efectos de producir conocimiento acerca de los procesos y dinámicas de la inclusión en su hacer(se) diario. A continuación, presentamos el enfoque metodológico que nos habilita problematizar la inclusión educativa en las escuelas seleccionadas.

\section{Metodología}

El presente artículo retoma el trabajo en terreno de tres investigaciones cualitativas (De Seena, 2015), que recupera aportes de la etnografía educativa (Rockwel y Anderson-Levitt, 2015), con foco en una analítica de las tensiones de la inclusión, en la cotidianeidad de escuelas emplazadas en contextos de pobreza urbana. En este artículo, entonces, trabajamos a partir de la noción de episodio (Armella y Grinberg, 2012; Darnton, 1987; Youdell, 2010) que se despliega en la vida cotidiana de las escuelas donde la inclusión se (auto)gerencia bajo el modo low cost (Bocchio, 2020). Ello en un marco, donde las escuelas de las periferias del sur global quedan inmiscuidas en las lógicas de empresas que brindan un servicio educativo (como el del transporte aéreo) y deben competir en una batalla desleal por obtener, retener y promocionar estudiantes y, al mismo, tiempo gestionar condiciones para sostener y hacer escuela. Estas escolarizaciones low cost son una de las principales barreras para que la inclusión escolar ocurra de forma masiva. Abordamos tales condiciones de escolarización a partir de tres episodios que se desarrollan en dos escuelas de la Provincia de Buenos Aires y en una de la Ciudad de Córdoba, Argentina.

La noción de episodio remite a un suceso que si bien puede leerse a simple vista como parcial sólo es dado a la comprensión si se lo integra en la historia. Esto es, como un evento singular y, a la vez, entramado en un todo que se nutre y complejiza. El análisis de episodios ha sido y es utilizado como herramienta metodológica en las investigaciones de aquella corriente que se denominó microhistoria ${ }^{2}$ en la segunda mitad del siglo XX, que comienza a centrar su atención en los sujetos y en el modo en que estos piensan el mundo, atendiendo no a grandes acontecimientos sino a pequeños e incluso extraños episodios de la vida cotidiana. Como señala Darnton (1987), partimos desde una microhistoria, donde se propone que estudiando un destino singular es posible conocer algunas características propias del mundo que lo circunda. Esta perspectiva tiene como foco de interés lo extraño, pero también lo cotidiano.

En el campo de la educación, el análisis de episodios ha sido trabajado por estudios etnográficos postestructuralistas, aunque aún sea un recurso escasamente explorado (Armella y Grinberg, 2012; Youdell, 2010). Es esa posibilidad de ubicar fragmentos o pequeños sucesos de la vida cotidiana de las escuelas en la historia, aquello que los convierte en un elemento sugerente que permite, asimismo, reconocer procesos de conformación, reconfiguración y pliegues en la subjetividad de quienes transitan territorios escolares abyectos como los que son aquí objeto de debate. Hablamos de instituciones donde los imperativos de la agenda política se topan con la precariedad como modo de hacer escuela a diario. Es así, también que trabajar aquí con tres episodios ocurridos en tres escuelas públicas de gestión estatal ofrece la oportunidad de comprender dinámicas, en este caso de inclusión-exclusión, que ocurren en esa lógica propia del siglo

2Influenciada por otras disciplinas como la antropología y la sociología encuentra entre sus principales referentes a Robert Darnton, Clifford Geertz, Carlo Ginzburg, Giovanni Levi, entre otros. 
XXI, donde lo local se vuelve global (Bauman, 2014). Ello porque se trata de escenas de la vida escolar que deben entenderse en las tensiones propias del sur global y a la vez en su particularidad y cotidianeidad para pensar ese inmunitas del que habla Espósito.

Es desde esta perspectiva que a continuación proponemos una caracterización de las tres escuelas para luego detenernos en los resultados de campo que, a modo de episodios, habilitan una analítica en torno a las formas de la inclusión escolar en estos espacios urbanos.

Escuela 1. Se trata de una escuela secundaria ubicada en una de las denominadas villas miseria o slums de la región metropolitana de Buenos Aires. Un territorio próximo al Río Reconquista, uno de los más contaminados del país, y de las áreas de relleno sanitario del CEAMSE, empresa que recoge y procesa los residuos de la Ciudad de Buenos Aires y del área metropolitana de la provincia de Buenos Aires. La basura se presenta en las vidas de quienes viven allí como desecho y a la vez como insumo, muchos de los vecinos son cartoneros y recuperadores urbanos.

Escuela 2. Se trata de una escuela de gestión estatal de educación especial para estudiantes con discapacidad intelectual emplazada en el primer cordón de la región metropolitana de Buenos Aires. La escuela es de jornada completa y cuenta con los servicios alimentarios para los casi 500 estudiantes que asisten a diario. Si bien esta escuela no se encuentra cercana a ninguna de las villas del Partido, recibe en un $60 \%$ a estudiantes que provienen de los barrios más empobrecidos de la ciudad emplazados a la rivera del Río mencionados con anterioridad. Sus familias viven en condiciones materiales de precariedad, con altas tasas de analfabetismo y desempleo estructural. La escena que describiremos se suscita en el patio de atrás de la escuela, que linda con otro patio de una escuela de educación común primaria. Ambas instituciones se emplazan en lo que se conoce como "manzana de las escuelas".

Escuela 3. La escuela secundaria se ubica en una Ciudad- Barrio ${ }^{3}$, al noreste de la Ciudad de Córdoba, los vecinos la bautizaron Barrio 29 de Mayo. Ciudad de Mis Cuartetos. Si bien el transporte público de colectivo ingresa a la Ciudad-Barrio por una calle sin pavimentar y en pésimo estado de mantenimiento, Ciudad de mis Cuartetos no tiene barrios aledaños, a diferencia de otros complejos habitacionales similares, está aislada. La escuela se encuentra ubicada en la parte más baja del complejo habitacional, por tanto, las cloacas en estado permanente de colapso atraviesan el ingreso a la escuela, el "paisaje" escolar también incluye un basural en la parte trasera de la escuela, que linda con el patio de recreo y el espacio al aire libre para educación física.

\section{Resultados}

Presentamos a continuación tres episodios seleccionados, específicamente, para abordar las condiciones contextuales y materiales en que la inclusión escolar se efectiviza en estas escuelas. En estos episodios, los equipos directivos de las tres escuelas se vuelven los y las protagonistas de escenas donde la materialidad de la pobreza y exclusión urbana atraviesa la escolaridad. Todas ellas son mucho más que metáforas, conforman las tramas de un

\footnotetext{
${ }^{3}$ La construcción de Ciudades- Barrios responde a una política habitacional impulsada en 2005 por el gobernador De la Sota para erradicar asentamientos y villas que estaban localizadas en zonas inundables de la ciudad (Bocchio,2019; Bocchio y Grinberg, 2019)
} 
hacer que, retomando a Esposito (2005), ocurre al calor del mandato de la inclusión mientras no dejan de ser parte de una negación que se hace cuerpo y cristaliza en cada rincón de las instituciones.

\subsection{Episodio 1. De directores (im)perfectos}

Estamos en viaje a la escuela emplazada en proximidad al Río Reconquista cuando Carlos, su director, nos llama para avisarnos que tienen que cerrarla. Están comunicándose con las familias para que pasen a retirar a cada estudiante.

Una vez más, se está inundando. Llueve hace días, pero ahora también "llueve desde abajo”, dice con voz de cansada resignación.

"Es un peligro, por las infecciones que puede dar esto. Pero el problema es que no saben cómo resolverlo porque no se encuentra la cámara séptica. $Y$ los chicos van a quedar de nuevo sin clases por varios días... o semanas. $Y$ ya vienen con muchas interrupciones, hubo jornadas en las que la falta de tubos de luz en las aulas hizo que no pudieran tener clase por horas, las aulas estaban prácticamente oscuras... y ìpodés creer que los tubos que nos mandaron desde el consejo escolar no los pudimos colocar porque tenían otra toma? A veces lamento ser tan torpe con mis manos. Porque si no me podría poner a arreglar y tal vez ganaríamos tiempo... $\Upsilon$ los chicos podrían tener clases antes" (Escuela 1. Registro de campo, agosto de 2019).

Carlos, en ese hacer encarnado en quien dirige una escuela en un asentamiento precario, como es su caso, ya no se queja de las condiciones de la infraestructura escolar, las conoce, las vive a diario y sabe que si quiere que la institución siga funcionando la única forma de torcerlas es actuando local y milimétricamente: ganar tiempo es la clave, siempre.

Aquí, en este segundo episodio las cañerías dan que hablar, atraviesan el día a día de la escuela y van más allá de sus paredes.

\subsection{Episodio 2. Encuentros orgánicos}

Es momento de recreo y salimos con el grupo y la docente al patio de atrás. La escuela de educación especial se encuentra inundada de intensos olores, entre ellos, los que provienen del desecho cloacal. Nos encontramos con la directora que, a la vez, es la encargada de uno de los grupos más pequeños debido a que la docente ese día se encuentra ausente por problemas de salud. En esos diez minutos que compartimos ese recreo sucede la siguiente conversación:

(Docente): De nuevo hay olor en la escuela, no se puede ni comer.

(Directora): Si, no te preocupes que ya me encargué de gestionar para que venga el Intendente a arreglar las cloacas. $Y$ como estamos en plena campaña electoral seguro nos cumplen el pedido.

(Investigadora): ¿Cómo hiciste?

(Directora): Callate que el sábado fui a la verdulería, me encontré a la mujer del intendente. Viste que viven por acá cerca. Me di cuenta que era ella y empecé a hablarle. Me dijo que llame al despacho y que hable para concertar una entrevista con el intendente y le cuente sobre el tema. Así que la semana que viene tenemos una reunión con el intendente [lo expresa entusiasmada y contenta].

(Docente): Que bueno. 
(Directora): Si, seguro vas vos y de paso le contas lo del trabajo que están haciendo sobre el cuidado del ambiente.

(Docente): Bueno dale ningún problema, todo sea para que nos pongan las cloacas.

(Directora): Si, no solo eso, hagamos una lista de todo lo que necesitamos, las cloacas, las veredas, la caldera, todo, así conseguimos la mayor cantidad de cosas y no nos olvidamos de nada. (Escuela 2. Registro de campo, septiembre de 2019)

Aprovechar la verdulería como lugar de reclamo, podría ser parte de una comedia de enredos, sin embargo, no lo es. Tampoco es la primera forma del reclamo, sino el modo en que la oportunidad hace al sujeto. Tampoco lo es la siguiente escena en donde la escuela debe acordar con la comisaria cómo hacer para que entren ambulancias a asistir a los estudiantes o limpiar un calabozo para que funcione de depósito de computadoras en el receso de verano. El siguiente episodio remite a ello.

\subsection{Episodio 3. El contrato es con el comisario.}

En una charla informal con la directora de la escuela secundaria de Ciudad de Mis Cuartetos nos comenta cómo resuelve problemas emergentes y cotidianos.

(Directora): Yo tengo un contrato con el comisario, eso me viene salvando.

Ante la imposibilidad de pagar un seguro médico en caso de accidentes en el espacio escolar (costo que -la directora aclara- las empresas privadas de asistencia a domicilio aumentan para no ingresar al barrio) el acuerdo es:

(Directora): Yo lo llamo al comisario, él llama el 107 y sacamos al pibe a la calle que es lugar donde el 107 tiene licencia para intervenir. (Escuela 3. Registro de campo, marzo 2020)

Al hablar de los escasos recursos con los que cuenta una escuela cuya especialidad es la de Artes Visuales, la directora comenta que hacia final de 2019 recibieron, por única vez, un carrito con netbooks provenientes del programa Aprender Conectados. Nuevamente el comisario aparece en escena y entre risas lo evoca.

(Directora): Directora le limpié el calabozo para que venga a guardar las computadoras por el verano. (Escuela 3. Registro de campo, marzo de 2020)

¿Qué elementos hilvanan esta serie de episodios? ¿Cuáles son las regularidades que hacen de ellos escenas singulares y a la vez capaces de narrar el presente en la cotidianeidad de las escuelas? ¿Qué dicen acerca de las formas que asume la inclusión educativa en el siglo XXI? Como señalamos, todos son episodios que en su narración resultan entre fantásticos o increíbles, e, incluso, podrían pensarse como situaciones de excepción. Sin embargo, se trata de escenas que atraviesan la cotidianeidad de las escuelas y por tanto permiten comprender mucho de la hechura de la escolaridad en estos espacios de la urbe. Una materialidad que explica mucho acerca de las condiciones en que se despliegan distintos procesos de inclusión escolar. A continuación, proponemos una analítica como un modo de aproximación a los clivajes de la inclusión gerenciada. Allí donde la desigualdad se combate a diario y su (auto)gestión se cristaliza en las más diversas estrategias de un hacer que, contraviento y marea, espera conseguir que algo del mandato de la inclusión se haga posible. 


\section{Discusiones y conclusiones}

$\mathrm{Al}$ comienzo del artículo planteamos como hipótesis que la inclusión educativa en escuelas, como las seleccionadas, signadas por múltiples desigualdades deviene inclusión gerenciada. De este modo hemos propuesto indagar las prácticas que signan el trabajo cotidiano en las instituciones escolares de directores y docentes. Prácticas que hacen de la inclusión un trabajo artesanal que supone administrar del mejor modo posible la precariedad que desborda, a diario, a las escuelas mientras procuran reponer condiciones mínimas que permitan a sus estudiantes estar en ella y que algo de lo escolar, allí, ocurra. Sin duda las escenas muestran de modo extremo esa inclusión-excluyente de la que habla Esposito (2005), una inclusión que no deja de negar la exclusión (segunda negación) en tanto la materialidad de la vida escolar no hace más que estallar. Las cañerías explotadas, como señalamos, son mucho más que una metáfora, se vuelven episodio. Esos episodios que requieren de la voluntad de los participantes se contraponen con los supuestos y los objetivos de la inclusión (Sandoval; Simón y Echeita, 2019; UNESCO, 2017) que es justamente, construir culturas, sociedades y escuelas más justas, más equitativas y menos excluyentes.

Ahora bien, en los episodios seleccionados se observa un plus que también es recurrente. Los tres directivos están cansados de esperar los tiempos del Estado y toman la iniciativa para "enmendar" la situación, hacen lo que pueden con los recursos que encuentran para resolver los problemas que surgen y urgen. Entonces ¿qué significa auto-gerenciar la inclusión? Podemos decir que son las maneras contemporáneas a través de las cuales se gestionan por cuenta propia las condiciones para ser y hacer escuela. Hablamos de condiciones de extrema desigualdad que obligan a directivos y docentes a auto-gestionarlo todo para que la escolarización de sus estudiantes sea posible. La inclusión así, en este tiempo y en estos contextos se vuelve una gesta individual. Una situación cuerpo a cuerpo para que las marcas de exclusión no dejen tanta huella.

En los casos seleccionados vemos sujetos trabajando en soledad. Los directores encuentran en algunos interlocutores extraescolares que no forman parte del sistema educativo. Ellos son la clave para resolver aquello que no está garantizado a través de los canales formales o previstos y que al mismo tiempo representan la posibilidad de conseguir lo necesario para el desarrollo de las clases. Estamos ante una serie de elementos que se repiten y articulan: la soledad de quienes cargan el peso de gestionarlo todo sin tener (casi) nada; el encuentro con interlocutores estratégicos (la mujer del intendente o el comisario) en los extramuros de la escuela (en la verdulería, en la comisaría) que permite que algo del intramuros escolar funcione.

Muchas de las cuestiones que retratan estos episodios refieren a la materialidad de las escuelas que de alguna manera se desploma ante nuestra presencia: cloacas que desbordan, falta de luz que posterga, por horas, el inicio de la clase, ausencia de espacios seguros para guardar recursos didácticos en la escuela, entre otros. Frente a esos escenarios la verdulería deviene lugar estratégico para pedir, a modo de favor y en tiempos electorales, que arreglen "la mayor cantidad de cosas" para que la institución pueda funcionar. Mientras tanto en otra de las escuelas la directora teje redes con el comisario a fin de tener un plan para cuando los estudiantes se accidentan o para cuidar del vandalismo los escasos recursos informáticos de los que disponen. Así, el policía le hace un favor a la directora y la mujer del intendente, inserta en la vorágine electoral, coordina una reunión con urgencia mientras compra en la verdulería. 
El mantenimiento de la cloaca no sería un problema si los directivos dispusieran de fondos de reparación, o una línea directa con el ministerio de educación para resolver el centenar de problemas emergentes; sin embargo, ni tales fondos llegan, ni la comunicación existe, de modo que la cloaca se transforma en un problema y, en la búsqueda de opciones para la resolución del mismo, el director "(im)perfecto" llega incluso a culparse por "ser tan torpe" con sus manos.

Estos episodios tienen, desde nuestra lectura, un hilo conductor que los aglutina: una materialidad atravesada por la ausencia y/o deficiencia de urbanización en la que están emplazadas las escuelas. Una muy particular forma que asumen las políticas de la vida urbana, las biopolíticas contemporáneas, que involucran unas condiciones de vida que dejan a vastas poblaciones arrojadas a condiciones de exposición diferencial a la violencia o a la contaminación, como es el caso de los barrios y escuelas de este estudio. Es en y a través de la gestión de esas condiciones que opera una inclusión que se vuelve excluyente (Mascareño y Carbajal, 2015).

Si bien consideramos que la inclusión, en estas condiciones, profundiza las desigualdades que procura suplir, planteamos que la autogestión de la inclusión, incluso ante este panorama hostil, acaba siendo, en la práctica, la única oportunidad para miles de estudiantes que sin ella quedarían incluso por fuera del sistema educativo.

Existen un sinfín de condiciones materiales y contextuales (carencia de recursos, basurales, cloacas estalladas) que sólo facilitarían la exclusión escolar si los sujetos representados en los tres episodios no fuesen el reflejo del modelo de director y directora que las escolarizaciones low cost demandan. Ellos dirigen escuelas que nunca están preparadas para dar clases, por tanto, es vital ganar tiempo a la precariedad estructural que signa sus condiciones de trabajo y las condiciones de aprendizaje de sus estudiantes. En ese ganar tiempo la autogestión es la respuesta hegemónica. Autogestión que, como mostramos también adquiere forma de gestión de oportunidades con el entorno próximo a la escuela para demandar todo tipo de recursos. La verdulería, la comisaría, el acto electoral, al aniversario de la escuela son oportunidades que hacen a los sujetos/directivos agentes de sus posibilidades de resolver problemáticas cotidianas.

La pregunta por la inclusión supone, ganar ese tiempo del que habla Carlos (Director de escuela 1), que se traduciría para Él en la posibilidad de tener clase. Más allá de cualquier agenda de política educativa y sus vaivenes, escuelas como las seleccionadas, escuelas que dan batalla, siguen siendo el cuarto intermedio en esa lucha que permite que los estudiantes estén en la escuela. Así, mientras estos episodios conforman parte clave del carácter aporético del procedimiento inmunitario al que refiere Esposito (2005), es quizá en ese siempre frágil equilibrio que la balanza cotidiana de las luchas también se escapa de él. La inclusión gerenciada es sin duda el engranaje clave de la gestión biopolítica de la vida (Foucault, 2013; Rose, 2007), pero, o quizá por eso, produce un plus en ese sostener a los estudiantes en la escuela a través de un sin fin de acciones que equipos directivos, docentes y padres llevan adelante de forma autónoma y solidaria para que el aprendizaje y la enseñanza tengan lugar (Bocchio y Villagrán, 2020). Es ese hacer escuela al que la lectura de los episodios remite, donde nos encontramos con un plus habilitado por el trabajar con otros, con la preocupación por el otro y con el otro.

Los tres episodios analizados plasman aquello que, entendemos, define condiciones y acciones elementales para que la inclusión pueda suceder. En otras palabras, cuando referimos a inclusión gerenciada hablamos de la búsqueda permanente de superar, en la 
lógica de la autoayuda, la carencia de condiciones para ser escuela que el Estado garantiza a los sectores "en riesgo de exclusión social y educativa". Queremos ser incisivas, el Estado en sus acciones produce efectos, en los episodios descritos se evidencia que, si las cloacas colapsan y no puede haber clases, esas son las condiciones reales de la materialidad estatal, aquellas que el Estado está administrando para garantizar la inclusión educativa. Esta es la cara más visible de la inclusión-excluyente, aquella "práctica homeopática de protección que excluye incluyendo y afirma negando" (Esposito, 2005, p. 18). Asimismo, entendemos que en las marcas que deja, algo del orden de lo no esperado también ocurre. Son las formas de las resistencias cotidianas que los episodios también expresan.

Ante estas determinantes lógicas de gobierno de la población, en las geografías descritas, la autogestión se mide en voluntad, una fuerza motriz poderosa, avasallante y peligrosamente neoliberal e individualista (Lopes y Fabris, 2016). En esa lógica, la sucesión de hechos que acontecen en las escuelas, cuando la inclusión-exclusión se produce, se asume la mayoría de las veces bajo el mandato de la escuela-empresa, del low cost (Bocchio, 2019). Hablamos de una inclusión gerenciada cuya precariedad cotidiana confirma, que, aun dotando a las escuelas de escasos recursos estatales el objetivo de escolarizar a quienes históricamente estuvieron fueran del sistema educativo se mantiene vigente. Como en las empresas low cost ellas, en sus huecos, habilitan la circulación de una población abyecta cuya sola presencia, no hace más que provocar. Y, en ella, en esa provocación descansan parte de las luchas contemporáneas que tienen a las escuelas como protagonistas clave. Cabe la posibilidad de abrir un interrogante ${ }^{\mathrm{S} S i}$ estos directivos no hiciesen este trabajo qué escolarizaciones tendríamos?

\section{Referencias}

Armella, J. y Grinberg, S. (2012). ¿Hay un hipertexto en esta clase? Dispositivos pedagógicos, tecnología y subjetividad. Signo Y Pensamiento, 31(61), 108-124. https://doi.org/10.11144/Javeriana.syp31-61.hhec

Bauman Z. (2013). Glocalization and hybridity, Glocalism: Journal of Culture, Politics and Innovation, $1,1-5$.

Bocchio, M. C. (2019). ¿Y si no estuviesen las escuelas secundarias en las Ciudades Barrios? Tensiones entre la mejora de las condiciones de escolarización y el desfinanciamiento de algunas políticas de inclusión socioeducativa en la Provincia de Córdoba. Archivos Analíticos de Políticas Educativas, 27(2)1-23. https://doi.org/10.14507/epaa.27.3178

Bocchio, M. C. (2020). Virajes en las políticas de inclusión social y educativa para garantizar la Educación Secundaria Obligatoria. Un estudio en ciudades-barrios de Córdoba, Argentina. Cuadernos de Humanidades, 31, 77-92.

Bocchio M. C. y Grinberg, S. (2019). Management times as moral regulation in Argentina. A study at the everyday work of a secondary school principal. International Journal of Leadership in Education, 22(1), 91-101. https://doi.org/10.1080/13603124.2018.1543539

Bocchio, M. C. y Villagrán, C. A. (2020) Regulación del espacio urbano, espacio escolar y dinámicas de solidaridad en la vida cotidiana. Estudios de caso en dos provincias argentinas. Espacios en Blanco. Revista de Educación, 30(2), 365-380. https://doi.org/10.37177/UNICEN/EB3O-283

Butler, J. (2010). Cuerpos que importan. Paidós.

Butler, J. (2006). Vida Precaria. El poder del duelo y la violencia. Paidós. 
Darnton, R. (1987). La gran matanza de gatos y otros episodios en la historia de la cultura francesa. FCE.

Duk, C. y Murillo, F. J. (2018). Una investigación inclusiva para una educación inclusiva. Revista Latinoamericana de Educación Inclusiva, 12(2), 11-13. https://doi.org/10.4067/So718-73782018000200011

Dussel, I. (2004). Inclusión y exclusión en la escuela moderna argentina: una perspectiva postestructuralista. Cadernos de Pesquisa, 34(122), 305-335. https://doi.org/10.1590/S0100-15742004000200003

Echeita. G. (2016). Educación para la inclusión o educación sin exclusiones. Narcea.

Esposito, R. (2005). Inmunitas: protección y negación de la vida. Amorrortu.

Etxeberria, X. (2018). Ética de la inclusión y personas con discapacidad intelectual. Revista Española de Discapacidad, 6(I), 281-290. https://doi.org/10.5569/2340-5104.06.01.14

Foucault, M. (2013). Nacimiento de la biopolítica. FCE.

Grinberg, S. (2019). Self-made school and the everyday making in Buenos Aires slums. British Journal of Sociology of Education, 40(4), 560-577. https://doi.org/10.1080/01425692.2019.1565991

Grinberg, S. (2020). Etnografía, biopolítica y colonialidad. Genealogías de la precariedad urbana en la Región Metropolitana de Buenos Aires. Tabula Rasa, 34, 19-39. https://doi.org/10.25058/20112742.n34.02

Infante, M. (2010). Desafíos a la formación docente: Inclusión educativa. Estudios Pedagógicos, 36, 1, 287-297. https://doi.org/10.4067/S0718-07052010000100016

Kaplan, K. (2017). La vida en las escuelas. Esperanza y desencantos de la convivencia escolar. Homo Sapiens.

Kessler, G. (2014). Controversias sobre la desigualdad. Argentina, 2003-2013. Fondo de Cultura Económica.

Mascareño, A. y Carvajal, F. (2015). Los distintos rostros de la inclusión y la exclusión. Revista Cepal, 116, 1331-146. https://doi.org/10.18356/087628b4-es

Lopes, M. C. y Fabris, E. T. (2016). Inclusão e educação. Autêntica.

Rockwell, E. y Anderson-Levitt, K. (2015). Importantes correntes de pesquisa etnográfica sobre educação: Maiorias, minorias emigrações através das Américas. Educação e Pesquisa, 41(2), 1129-1135. https://doi.org/10.1590/S1517-9702201508148880

Rose, N. (2007). The politics of life itself. Princeton University Press. https://doi.org/10.1515/9781400827503

Sandoval, M. Simón, C. y Echeita, G. (2019). Educación inclusiva y atención a la diversidad desde la orientación educativa. Pirámide.

UNESCO. (2017). Guía para asegurar la equidad y la inclusión en la educación. UNESCO.

Valls, J. F. (2013). Más allá del "lowcost”, productos, precios y marcas en el nuevo escenario de la sensibilidad al precio. Asociación para el Progreso de la Dirección, 283, 14-16.

Youdell, D. (2010). Queer outings: Uncomfortable stories about the subjects of poststructural school ethnography. International Journal of Qualitative Studies in Education, 23(1), 87-100. https://doi.org/10.1080/09518390903447168 


\section{Breve CV de las autoras}

\section{$\mathrm{M}^{\mathrm{a}}$ Cecilia Bocchio}

Doctora en Educación. Doctorado en Política y Administración Educativa. Universidad de Lisboa, Becaria Eramus-Mundus Unión Europea. Licenciada y Profesora en Ciencias de la Educación. Universidad Nacional de Córdoba-Argentina. Investigadora asistente del Consejo Nacional de Investigaciones Científicas y Técnicas (CONICET). Profesora Asistente Regular de Política Educacional y Legislación Escolar, Licenciatura en Ciencias de la Educación, FFYH, UNC. ORCID ID: https://orcid.org/O000-0003-3915-6857. Email:mcbocchio@gmail.com

\section{Cintia Schwamberger}

Licenciada y Profesora de Educación Especial (UNSAM). Becaria Doctoral del Consejo de Investigaciones Científicas y Técnicas (CONICET), en el Laboratorio de Investigación en Ciencias Humanas (LICH). Miembro del equipo de Investigación CEDESI EHUUNSAM. Miembro investigador del Grupo de Trabajo en Estudios Críticos de la Discapacidad (CLACSO). Docente de la Escuela de Humanidades de la UNSAM. ORCID ID: https://orcid.org/0000-0002-2409-0851. Email: cintiaschwamberger@gmail.com

\section{Julieta Armella}

Doctora de la Universidad de Buenos Aires, área Ciencias de la Educación. Investigadora asistente del Consejo Nacional de Investigaciones Científicas y Técnicas (CONICET). Miembro del Laboratorio de Investigación en Ciencias Humanas (LICH) del Centro de Estudios en Desigualdades, Sujetos e Instituciones (CEDESI). Docente de la Escuela de Humanidades UNSAM, en Sociología de la educación. ORCID ID: https://orcid.org/0000-0002-2401-032 1. Email: juli.armella@gmail.com

\section{Silvia Grinberg}

Doctora en Educación por la Universidad de Buenos Aires (UBA) y magíster en Ciencias Sociales por la Facultad Latinoamericana de Ciencias Sociales (FLACSO). Es investigadora del Consejo Nacional de Investigaciones Científicas y Técnicas (CONICET), directora del Laboratorio de Ciencias Humanas LICH-UNSAM-CONICET y del Centro de Estudios en Desigualdades, Sujetos e Instituciones (Cedesi) y profesora en la Escuela de Humanidades de la Universidad Nacional de San Martín (UNSAM), Argentina. ORCID ID: https://orcid.org/O000-0001-9261-9035 Email: grinberg.silvia@gmail.com 